\title{
Molecular Mechanism of Enhanced Anticancer Effect of Nanoparticle Formulated LY2835219 via p16-CDK4/6-pRb Pathway in Colorectal Carcinoma Cell Line
}

\author{
Xu Tang, ${ }^{1}$ Bin Zeng, ${ }^{2}$ Jian-Kun Gao, ${ }^{3}$ and Han-Qiang Liu ${ }^{4}$ \\ ${ }^{1}$ Department of Pathology, Sichuan College of Traditional Chinese Medicine, Mianyang, China \\ ${ }^{2}$ Department of Pharmacy and Inspection, Sichuan College of Traditional Chinese Medicine, Mianyang, China \\ ${ }^{3}$ Department of Basic Medical Sciences, Sichuan College of Traditional Chinese Medicine, Mianyang, China \\ ${ }^{4}$ Department of Nutrition and Food Hygiene, The Fourth Military Medical University, Xian 710032, China
}

Correspondence should be addressed to Han-Qiang Liu; liuhanqiangvip@163.com

Received 5 May 2016; Accepted 15 June 2016

Academic Editor: Mingqiang Li

Copyright (C) 2016 Xu Tang et al. This is an open access article distributed under the Creative Commons Attribution License, which permits unrestricted use, distribution, and reproduction in any medium, provided the original work is properly cited.

\begin{abstract}
LY2835219 is a dual inhibitor to CDK4 and CDK6. This study was to prepare LY2835219-loaded chitosan nanoparticles (CNP/LY) and LY2835219-loaded hyaluronic acid-conjugated chitosan nanoparticles (HACNP/LY) and revealed their anticancer effect and influence on p16-CDK4/6-pRb pathway against colon cell line. The nanoparticle sizes of CNP/LY and HACNP/LY were approximately $195 \pm 39.6 \mathrm{~nm}$ and $217 \pm 31.1 \mathrm{~nm}$, respectively. The zeta potentials of CNP/LY and HACNP/LY were $37.3 \pm 1.5 \mathrm{mV}$ and $30.3 \pm 2.2 \mathrm{mV}$, respectively. And the preparation process showed considerable drug encapsulation efficiency and loading efficiency. LY2835219, CNP/LY, and HACNP/LY inhibited HT29 cell proliferation with $0.68,0.54$, and $0.30 \mu \mathrm{M}$ of IC50, respectively. G1 phase was arrested by LY2835219 and its formulations. Furthermore, inhibition of CDK4/6 by LY2835219 formulations induced CDK4, CDK6, cyclin D1, and pRb decrease and p16 increase at both protein and mRNA levels. Overall, nanoparticle formulated LY2835219 could enhance the cytotoxicity and cell cycle arrest, and HACNP/LY strengthened the trend furtherly compared to CNP/LY. It is the first time to demonstrate the anticancer effect and mechanism against HT29 by LY2835219 and its nanoparticles. The drug and its nanoparticle formulations delay the cell growth and arrest cell cycle through p16-CDK4/6-pRb pathway, while the nanoparticle formulated LY2835219 could strengthen the process.
\end{abstract}

\section{Introduction}

Colorectal cancer (CRC) is one of the most frequent human cancers with the third highest mortality rate in current cancer mortality statistics in the European Union (EU) [1]. Chemotherapy is a common treatment with considerable side effects, such as drug distribution on normal cells. Drug delivery system (DDS) can target colon tumor cells and enhance the uptake of anticancer drugs inside tumors [2].

Chitosan is natural, nontoxic, biocompatible, and biodegradable with wide application in DDS. Due to its mucoadhesive properties, chitosan is good material forming nanoparticles (NPs) for targeting colorectal cancer. Numerous reports have confirmed that chitosan can prolong the retention time in the locations of interest and increase the cellular uptake and tumor accumulation of NPs [3-5]. Hyaluronic acid (HA), a drug carrier, is nonantigenic and biocompatible, possessing strong affinity toward the CD44-overexpressing cells. It was reported that $\mathrm{CD} 44$ receptor was overexpressed in multiple human cancer cells. Therefore, NPs with HA conjugation are expected to have great potential in clinical applications [6]. NPs based on chitosan or HA have been applied to colontargeted delivery [7-11].

The study of underlying pathological mechanisms of cancer is important to improve the cure rate and drug research. Cell cycle is a vital mechanism for tumor development and malignance [12], and p16-CDK4/6-pRb pathway is the most important regulatory signaling pathway involved in the G1/S transition. Retinoblastoma $(\mathrm{Rb})$ is phosphorylated to trigger the transition by kinase of CDK4 or CDK6 with cyclin D1 [13] . 
Many studies have proved that cyclin D1-CDK4/6 complex is overexpressed in human tumor tissues. Thus, CDK4 or CDK6 plays a critical role for checkpoint regulation. p16 is a member of cyclin-dependent kinase inhibitor protein (CKI), which inhibits CDKs in order to negatively control cell cycle. Excessive tumor cell growth was usually promoted by destruction of cell cycle, such as abnormal overexpression of the cyclin D1-CDK4/6 complex and phosphorylation of Rb, as well as a low level of p16. Abnormalities of p16-CDK4/6-pRb pathway exist in a variety of malignances including colorectal cancer, breast cancer, lung cancer, head and neck cancer, and gastric cancer [14].

LY2835219, a selective dual inhibitor to CDK4 and CDK6, shows promise for non-small cell lung cancer (NSCLC) and breast cancer in phase I study [15]. Tate et al. [16] reported that LY2835219 mediated CDK4/6 inhibition, cell cycle arrest, and tumor growth inhibition in colo-205 human colorectal xenografts. Although pRb formation and CDK4/6 in tumors were successfully inhibited in vivo, the reports on the in vitro efficacy and pharmacological studies on colon cell lines are few.

Based on the integration advantages of drug with DDS, in this study, chitosan and HA were used to prepare the NPs to load LY2835219. It was the first time for colon cell line that the anticancer effect and mechanism of LY2835219 and its nanoparticle formulations were evaluated. The effect on p16-CDK4/6-pRb pathway was elucidated as the mechanism study.

\section{Materials and Methods}

2.1. Preparation of LY2835219-Loaded NPs. Chitosan was dispersed into the acetic acid $(0.1 \mathrm{M}, \mathrm{pH} 4.0)$ and stirred to obtain a transparent solution $(2 \mathrm{~g} / \mathrm{L})$ and then filtered through a $0.22 \mu \mathrm{m}$ membrane. LY2835219 (Selleck) was dissolved into TPP aqueous solution $(1 \mathrm{~g} / \mathrm{L})$ to make a final concentration of $100 \mathrm{~g} / \mathrm{L}$. Chitosan solution (5 mL) and LY2835219 solution $(2 \mathrm{~mL})$ were mixed with vigorous stirring to form a milky mixture. The suspensions were centrifuged for $30 \mathrm{~min}$ at $10,000 \mathrm{rpm}$. The sediments were dialyzed three times against phosphate buffered saline (PBS, pH 7.4) for $30 \mathrm{~min}$, followed with lyophilization to form LY2835219-loaded chitosan NPs (CNP/LY).

Before conjugation of HA to CNP/LY, HA was dissolved in $\mathrm{PBS}$ and stirred for $60 \mathrm{~min}$. Lyophilized CNP/LY was suspended into HA solution with the CNP/LY:HA ratio of $10: 1$ in the presence of $\operatorname{EDC}(20 \mathrm{~g} / \mathrm{L})$ for the aim of cross-link and then vortexed for $60 \mathrm{~min}$. The HA-conjugated CNP/LY was filtered, collected, and washed three times using a Sephadex G-75 spin column by centrifugation. The filtrate was lyophilized and named as LY2835219-loaded HAconjugated chitosan nanoparticles (HACNP/LY).

2.2. Measurement of Characteristics of LY2835219 Formulations. The drug encapsulation efficiency (EE) and loading efficiency (LE) were calculated to present the efficiency of NPs production. LE was defined as the ratio of the mass of LY2835219 in NPs to the total mass of NPs, and EE was the percentage of the mass of LY2835219 to the mass of the total used LY2835219 for NPs preparation. The mass and purity of LY2835219 in NPs were measured by HPLC. The important parameters of HACNP/LY and CNP/LY, such as the average size and zeta potential, were investigated by Zetasizer Nano $S$ (Malvern Instruments, UK). All measurements were performed in triplicate.

2.3. In Vitro Drug Release Studies. The purpose of this assay was to investigate the release characteristics of HACNP/LY and CNP/LY. The studies were conducted using dialysis bags soaking in medium at physiological $\mathrm{pH}(\mathrm{pH}$ 7.4) and in the presence of serum to simulate in vivo conditions. The dialysis bag containing $1 \mathrm{mg}$ of drug-loaded NPs suspending in $2 \mathrm{~mL}$ of release media (DMEM containing $10 \%$ fetal bovine serum) was placed in a beaker with $20 \mathrm{~mL}$ of release media. The dialysis was maintained at $37^{\circ} \mathrm{C}$ for 3 days with stirring at $100 \mathrm{rpm}$. Periodically $1 \mathrm{~mL}$ of samples in beaker was collected and the same volume of fresh media was added. The concentration of drug was quantified using HPLC.

2.4. Cell Culture. HT29, human colorectal carcinoma cell line, was purchased from ATCC. HT29 was cultured in DMEM (HyClone) with 10\% fetal bovine serum (Life Technologies) and $1 \%$ penicillin-streptomycin in an atmosphere of $5 \% \mathrm{CO}_{2}$ at $37^{\circ} \mathrm{C}$.

2.5. Cell Growth Inhibition Assay. CCK-8 (Dojindo, Japan) assay was used for measuring the antiproliferation of LY2835219 and its formulations. Briefly, HT29 cells were trypsinized to prepare the single-cell suspensions. Cells were counted and added to 96-well plate $(2000 /$ well/100 $\mu \mathrm{L})$, followed with 3-fold serial dosage of LY2835219 or its formulations. After $72 \mathrm{~h}$ treatment, $10 \mu \mathrm{L}$ of CCK- 8 was added to each well and the absorbance at $450 \mathrm{~nm}$ was measured according to the manufacturer. IC50 was calculated by GraphPad Prism software.

2.6. Real-Time Quantitative PCR ( $q R T-P C R)$. Cells were harvested after treatment with $0.1 \mu \mathrm{M}$ of LY2835219 and its formulations for 72 hours. Medium was used as the negative control. TRIzol reagent (Invitrogen) was used to extract total RNA from the cells. Complementary DNA (cDNA) was synthesized using QuantScript RT Kit (Qiagen) according to manufacturer's instruction. Random primers were used for the reverse transcription. qRT-PCR protocol for assessment of RNA quantity was assayed by the ABI 7500 (Applied Biosystems) and conducted in 96-well optical reaction plates with a final volume of $50 \mu \mathrm{L}$ using Real Master Mix (Qiagen). GAPDH was used as an internal reference gene for normalization. The reverse primers were designed with Primer Express 3.0 software. The PCR primer sequences were as follows: CDK4 (forward 5'-GCC CAG AAC GTC CGG C$3^{\prime}$; reverse $5^{\prime}$-TCC TAC GGC CCC ATA CAC C-3'), CDK6 (forward $5^{\prime}$-CTG ACA CTC GCA GCC CC-3'; reverse $5^{\prime}$ CCT CCT CTT CCC TCC TCG AA- $3^{\prime}$ ), p16 (forward $5^{\prime}$ GAT TTG AGG GAC AGG GTC GG-3'; reverse $5^{\prime}$-CCA GCC AGT CAG CCG AAG-3'), Rb (forward $5^{\prime}$-CAG GCT TGA GTT TGA AGA AAC AGA- $3^{\prime}$; reverse $5^{\prime}$-AGA TAC AGA TTC CCC ACA GTT CC- $3^{\prime}$ ), and GAPDH (forward 
TABLE 1: Characterization of LY2835219-loaded nanoparticles (mean $\pm \mathrm{SD}, n=3$ ).

\begin{tabular}{lcccc}
\hline Formulation & LE $(\%)$ & EE $(\%)$ & Particle size $(\mathrm{nm})$ & Zeta potential $(\mathrm{mV})$ \\
\hline CNP/LY & $21.3 \pm 5.2$ & $41.7 \pm 7.1$ & $195 \pm 39.6$ & $37.3 \pm 1.5$ \\
HACNP/LY & $24.1 \pm 3.5$ & $36.9 \pm 6.2$ & $217 \pm 31.1$ & $30.3 \pm 2.2$ \\
\hline
\end{tabular}

$5^{\prime}$-TTG CCC TCA ACG ACC ACT TT-3'; reverse $5^{\prime}$-CCA CCA CCC TGT TGC TGT AG-3' ${ }^{\prime}$.

2.7. Flow Cytometric Analysis of Cell Cycle. Cells were plated in 24-well plate $\left(5 \times 10^{4}\right.$ cells/well $\left./ 1 \mathrm{~mL}\right)$ overnight, followed by incubation with IC50 concentration of drug and drug formulations for $24 \mathrm{~h}$, and then they were harvested by trypsinization and fixed with $4 \%$ paraformaldehyde for $15 \mathrm{~min}$. The cell pellet was incubated in a solution containing $50 \mu \mathrm{g} / \mathrm{mL}$ propidium iodide (PI), $0.1 \mathrm{mg} / \mathrm{mL}$ RNase, and $0.05 \%$ Triton $\mathrm{X}-100$ at $37^{\circ} \mathrm{C}$ for $30 \mathrm{~min}$. The cells were analyzed by flow cytometer (BD, FACSCalibur).

2.8. Western Blot Assay. HT29 cells were treated with $0.1 \mu \mathrm{M}$ of LY2835219 and its NPs for $24 \mathrm{~h}$. Then proteins were detected by Western blot for CDK4, CDK6, cyclin D1, p16, and $\mathrm{pRb}$ expressions. Briefly, cell lysates were prepared in cell lysis buffer (Sigma) containing $1 \mathrm{mM}$ PMSF. The total protein content was measured by Coomassie brilliant blue assay (Beyotime). The equal amount of protein was electrophoresed on 10\% SDS-PAGE and transferred to PVDF membranes. The membranes were blocked, incubated for $2 \mathrm{~h}$ with primary antibodies, and subsequently incubated with secondary antibodies (Boster) for $2 \mathrm{~h}$. The blots were visualized using ECL detection reagents (Beyotime). The following antibodies were used: CDK4, CDK6, and cyclin D1 from Beyotime, p16 and pRb from Boster, and GAPDH from Abcam.

2.9. Statistical Analysis. All statistical analyses were performed by the SPSS 19.0 software package (SPSS Inc.). Student's $t$-tests were used for comparison between independent groups.

\section{Results and Discussion}

3.1. Preparation and Characterization of NPs. In this study, HACNP/LY was prepared and applied to improve the anticancer efficacy of the anticancer drug, LY2836219. The drug was fabricated with chitosan by TPP ionic cross-linking [17, 18]. And the CNP/LY was modified by the covalent reaction between amino groups of chitosan and the carboxy groups of HA $[19,20]$. The LE and EE revealed the production efficiency. LY2835219 was loaded into the core of chitosan with reasonable efficiency. The LE of CNP/LY and HACNP/LY was $21.3 \pm 5.2 \%$ and $24.1 \pm 3.5 \%$, respectively (Table 1 ). The $\mathrm{EE}$ of $\mathrm{CNP} / \mathrm{LY}$ was $41.7 \pm 7.1 \%$ and lower $\mathrm{EE}$ value was obtained when HA conjugated to chitosan with the value of $36.9 \pm 6.2 \%$. These results can be explained due to the loss of the drug material during the encapsulation procedure. The average size of CNP/LY was found to be around

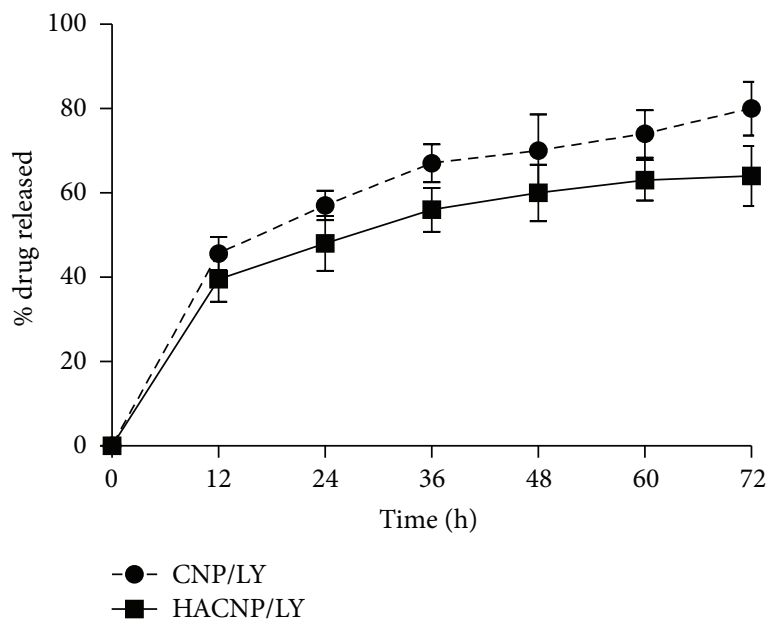

FIGURE 1: In vitro cumulative release (\%) study of LY2835219 from HACNP/LY or CNP/LY in physiological $\mathrm{pH}(\mathrm{pH} 7.4)$ media at $37^{\circ} \mathrm{C}$ by dialysis membrane assay.

$195 \pm 39.6 \mathrm{~nm}$, while conjugated HA tended to increase the particle size into $217 \pm 31.1 \mathrm{~nm}$. The zeta potential of $\mathrm{CNP} / \mathrm{LY}$ and HACNP/LY was observed to be $37.3 \pm 1.5$ and $30.3 \pm 2.2 \mathrm{mV}$, respectively. The decreased value of zeta potential may be due to the surface cover on positive charges of chitosan by negative charges of HA.

3.2. NPs Made from Chitosan and HA Could Slow Down the LY2835219 Release. As shown in Figure 1, a rapid release presented within the first $12 \mathrm{~h}$ of both drug-loaded NPs, but when release went on, the slopes of curve trended to gentle. Two LY2835219 formulations had similar release patterns. It was speculated that the rapid release within the first $12 \mathrm{~h}$ might be due to the weak adhesion of LY2835219 on the NPs surface. Accompanying with the water absorption and swelling of chitosan, the hydrophilic gel layer is formed eventually in the surface of nanoparticles, which shut down the drug release channel, to slow down the release of LY2835219 through spread or dissociation on account of degradation of nanoparticles. Overall, the release of HACNP/LY performed more slowly than that of CNP/LY. It suggested possibly the water absorption and swelling of HA stopped and delayed the release which resulted in a slow speed.

3.3. Nanoparticle Formulations Enhance the Cytotoxic Ability of LY2835219 against HT29 In Vitro. The in vitro potencies of different LY2835219 formulations were tested by CCK-8 assay. As shown in Figure 2, for LY2835219 or any formulations, the inhibition against HT29 proliferation was presented in a dose-dependent manner. As expected, the IC50 of CNP/LY $(0.52 \pm 0.112 \mu \mathrm{M})$ was more potent than that 


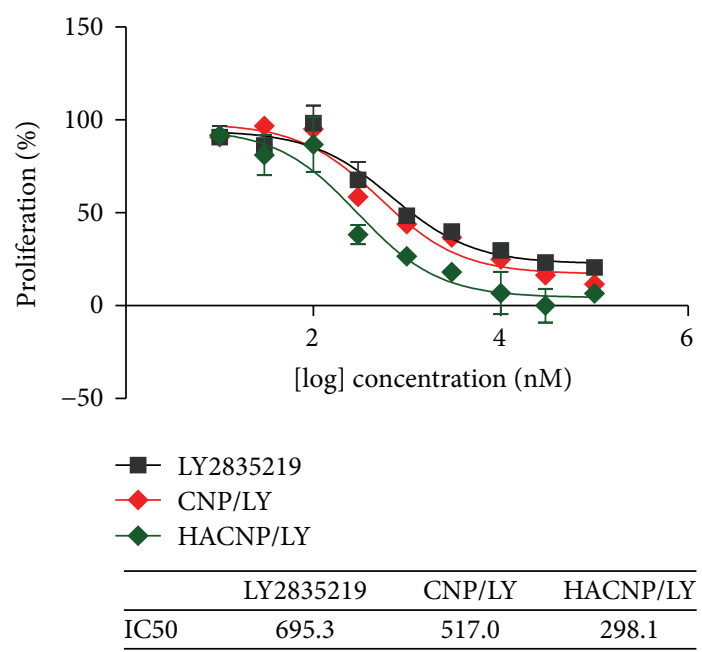

FIGURE 2: Antiproliferation effect on colorectal carcinoma cells in a dose-dependent manner by CCK- 8 assay.

of LY2835219 $(0.68 \pm 0.094 \mu \mathrm{M})$. Furthermore, HACNP/LY $(0.30 \pm 0.077 \mu \mathrm{M})$ exhibited near 2-fold potent activity compared to that of CNP/LY. It indicated that the CDK4/6 inhibitor had the antiproliferation activity against colorectal carcinoma cell line. Additionally, the increased potency by NPs suggested that chitosan was effective to deliver drug into target cell, and HA enhanced the ability of chitosan.

To explain the gradually potent activities by LY2835219, $\mathrm{CNP} / \mathrm{LY}$, and HACNP/LY, it might be attributed to stronger negative charges on the cancer cells compared to normal cells due to universal existence of human chorionic gonadotropin on the surface of cancer cells, which contained large amount of negative charges [21]. Thus, the positive surface charges on chitosan can enhance the selective cellular uptake and anticancer efficacy [17]. On the other hand, the cytotoxicity may be due to the direct inhibition of chitosan against cancer cells [22]. Additionally, the enzymatic production of chitosan, oligosaccharides (COS), also reported the anticancer efficacy, while it had no effect on normal cells [23]. Hyaluronic acid is a natural biopolymer found in many tissues of the human body and is responsible for various physiological functions [24]. Notably, HA functions interrelate with the primary receptors of HA, CD44, RHAMM, LYVE-1, and HARE [25]. Among these receptors, CD44 is reported to be highly expressed on breast, ovarian, lung, and colorectal cancer [21, 26]. According to the receptor-ligand binding theory, HA can bind with CD44 on the surface of cancer cells. Based on this rationale, HA-conjugated NPs can deliver anticancer agent into cancer cells with higher efficiency.

3.4. Cell Cycle Arrest at G1 Phase by LY2835219 and Its Nanoparticle Formulations on HT29 Cells. As shown in Figures 3(a) and 3(b), the cell cycle of HT29 cells was changed by LY2835219 and its nanoparticle formulations. Compared to LY2835219-free group, the number of cells in the G1 phase increased from $35.89 \pm 1.69 \%$ to $45.54 \pm 2.9 \%$ in HT29 cells. Similarly, cell number in the G1 phase increased significantly to $49.40 \pm 3.82 \%$ and $56.33 \pm 4.22 \%$ by CNP/LY and
HACNP/LY, respectively, compared to either control group or LY2835219 group. The progression of cell cycle was arrested at G1 phase.

The cytotoxicity demonstrated the anticancer effect of LY2835219 and its formulations, and the cell cycle was furtherly analyzed for the mechanism study purpose. In this study, G1 cell cycle arrest verified that the CDK4/6 activity was inhibited by LY2835219 and the result was similar to LY2835219 inhibition on other cancer cells [26]. Furtherly, drug NPs loaded by chitosan or HA-conjugated chitosan could notably enhance this $\mathrm{Gl}$ arrest. And cancer cell targeted HA could enhance this trend mostly.

3.5. Effects of Different LY2835219 Formulations on mRNA Expression of CDK4/6 Relevant Signaling Molecules. qRTPCR reveals the changes of upstream and downstream mRNA of CDK4/6 expressed in HT29. Firstly, observed expression of CDK4 and CDK6 mRNA verified the effective possibility of CDK4/6 inhibitor, as well as the necessity for further study (Figure 4(a)). Moreover, after $72 \mathrm{~h}$ treatment of different LY2835219 formulations on cells, the mRNA level of p16 (the upstream molecule) increased significantly, whereas expression of $\mathrm{Rb}$ (the downstream molecule) decreased significantly (Figure 3(b)). This result proved that LY2835219 could affect the upstream and downstream gene expressions of CDK4/6, and the NPs had the ability to enhance the effect.

3.6. LY2835219 Formulations Affect the Protein Expression of CDK4/6 and Its Relevant Proteins in Its Signal Pathway. As shown in Figure 5, CDK4 and CDK6 protein expression decreased by their inhibitor, LY2835219, as well as its formulations. Cyclin D1, the partner of CDK4 and CDK6, decreased too. p16 and pRb, the upstream and downstream molecules of CDK $4 / 6$, increased and decreased, respectively. It indicated that inhibition to CDK4/6 had an effect on the protein expression of their upstream and downstream molecules and their partner molecule. Furthermore, nanoparticle formulations could enhance this effect, and HACNP/LY enhanced the trend more notably.

The changes of mRNA and protein level of CDK4/6 and their relevant molecules could reveal changes in the signaling pathway. As CDK4/6 dual inhibitor, LY2835219 showed promising effect against NSCLC and breast cancer in phase I study [15]. p16-cyclin D1/CDK4/6-pRb signaling pathway was an important pathway in the study of CDK4 and CDK6 inhibitors. Thus, the influence on this pathway by different LY2835219 formulations was studied.

Destruction of cell cycle usually promotes excessive tumor cell growth. And cell cycle destruction maybe comes from abnormal overexpression of the cyclin D1-CDK4/6 complex, a low expression of CKI, or inactivation of $\mathrm{Rb}$. Abnormalities of p16-cyclin D1/CDK4-pRb pathway exist in a variety of malignances including breast cancer, lung cancer, head and neck cancer, gastric cancer, intestinal cancer, and malignant lymphoma. Relationships between clinical and pathological features were studied [14]. In that study, the molecule expressions in p16-cyclin D1/CDK4-pRb pathway were detected in 67 resected gastric adenocarcinomas and 

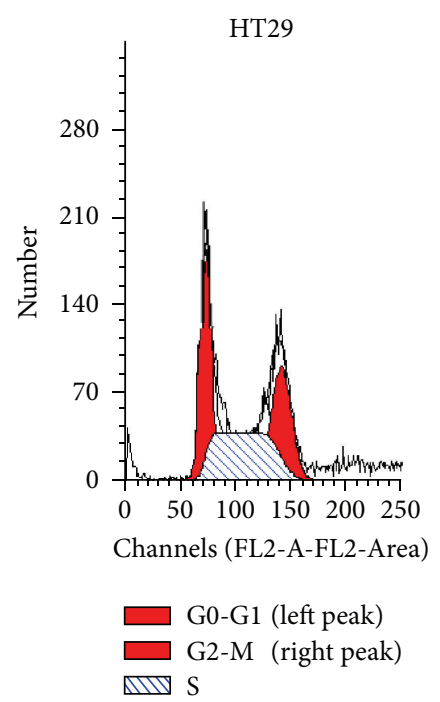

MIV $S$
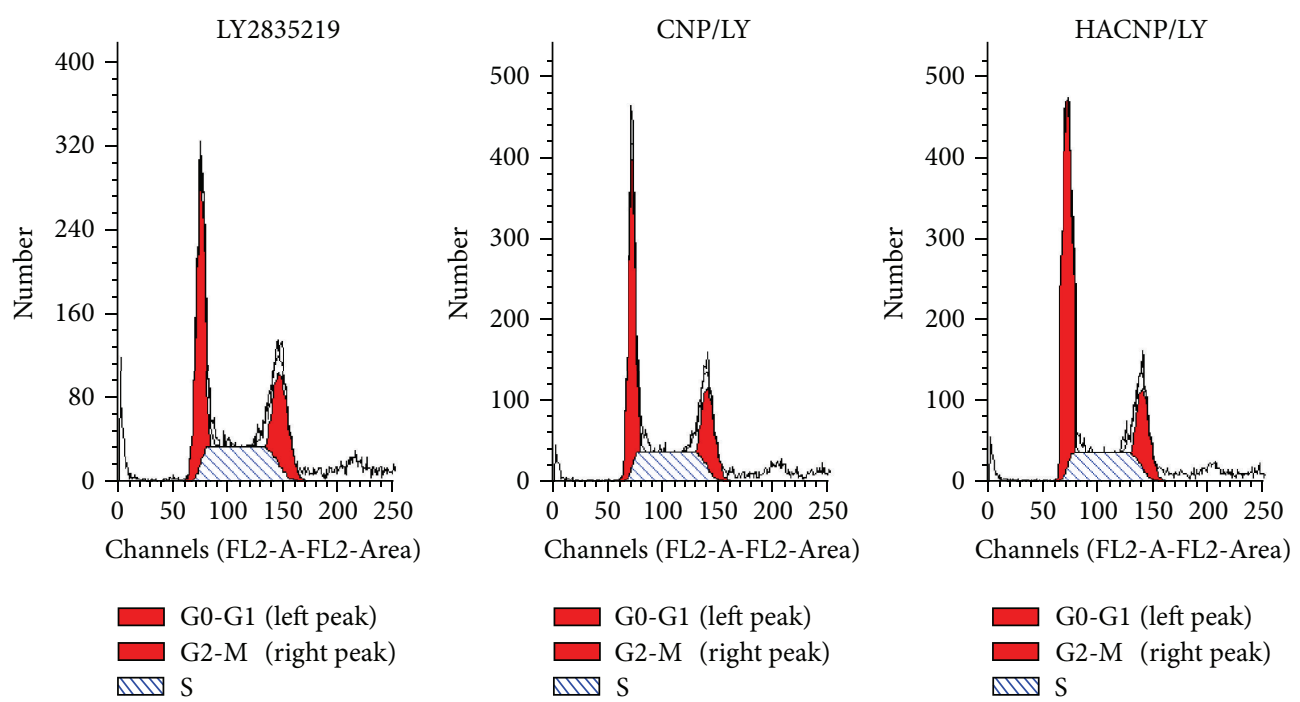

NIIV $\mathrm{S}$

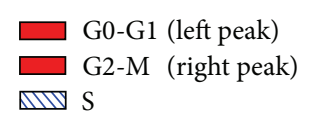

(a)

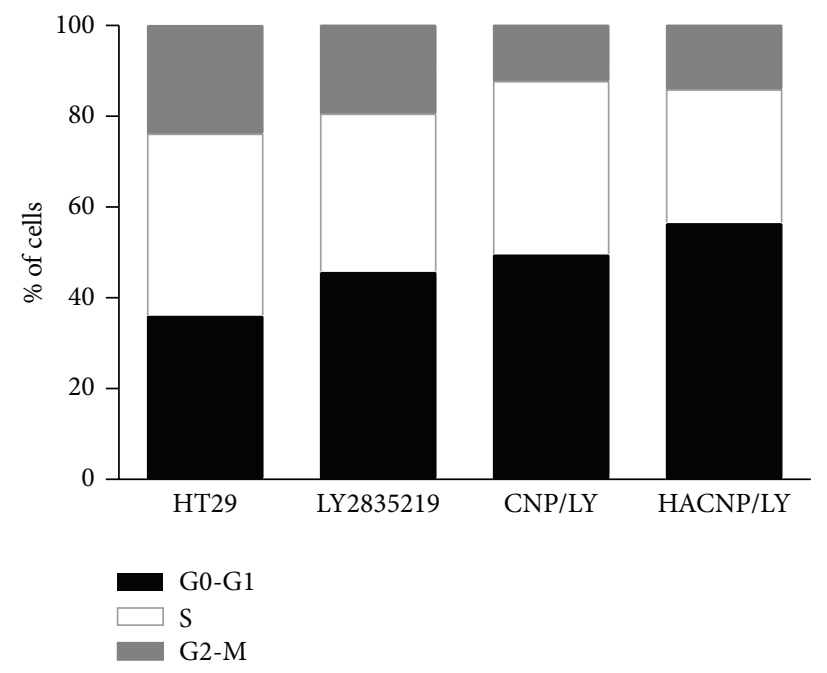

(b)

Figure 3: Cell cycle arrest at G1 phase by LY2835219 and its nanoparticle formulations.

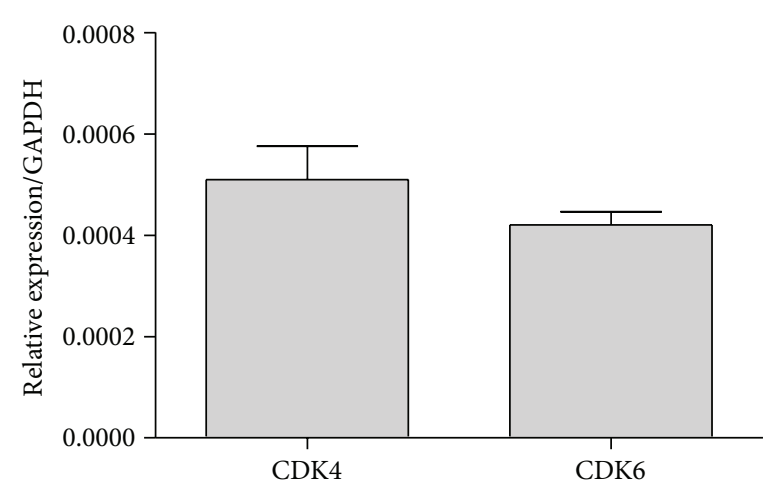

(a)

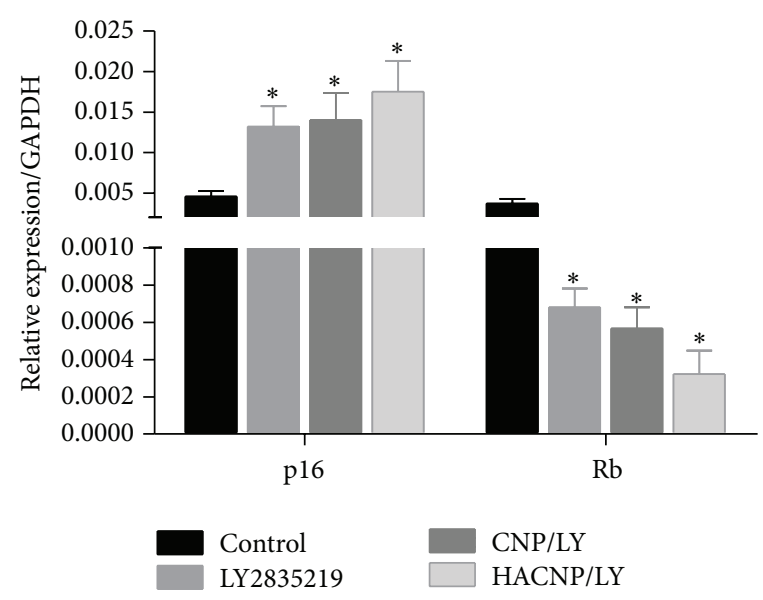

(b)

FIGURE 4: Effects of LY2835219 and its nanoparticle formulations on gene expression of CDK4/6 relevant signaling molecule by qRT-PCR. ${ }^{*} P<0.05$ compared with the CIA group. 


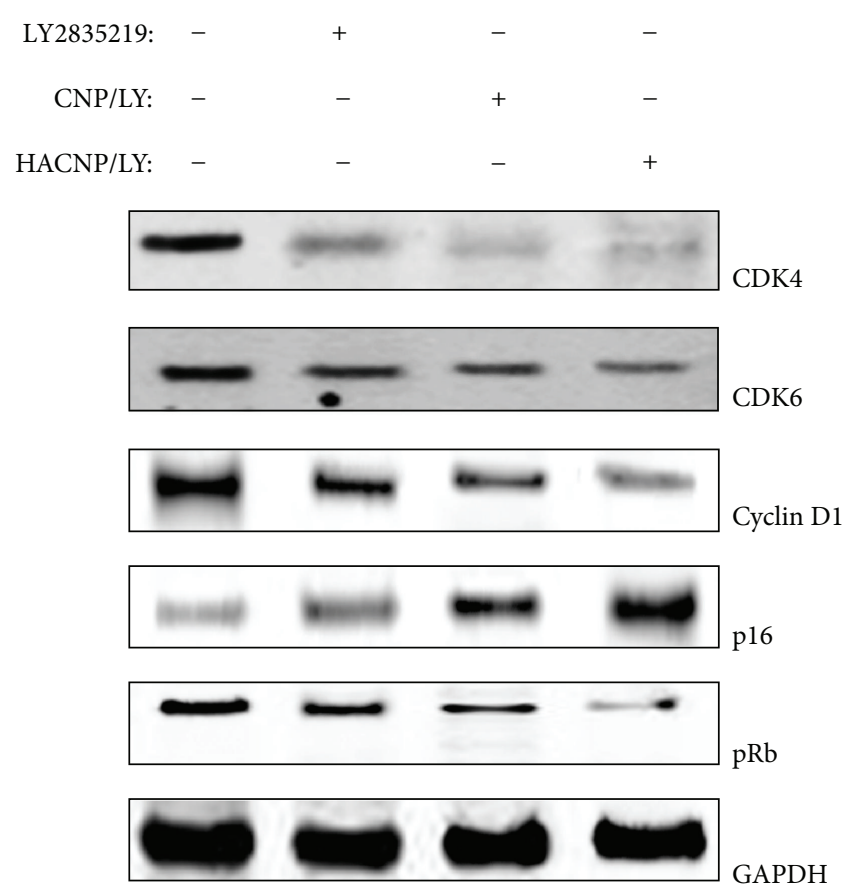

(a)

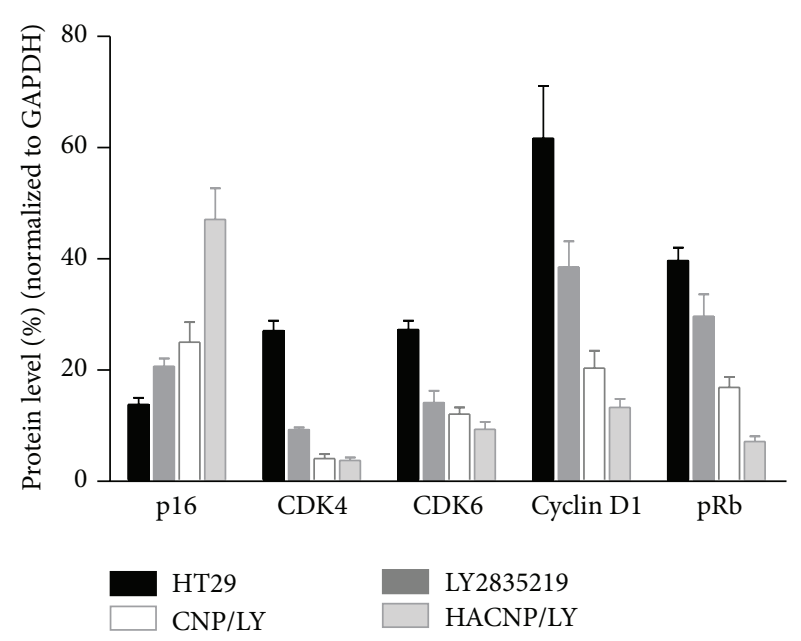

(b)

FIGURE 5: LY2835219 and its nanoparticle formulations have the effect on the protein expression of CDK4/6 and its relevant proteins. (a) Western blotting analysis. (b) Densitometry performed for the different protein and normalized to GAPDH. $n=3$, mean \pm SD.

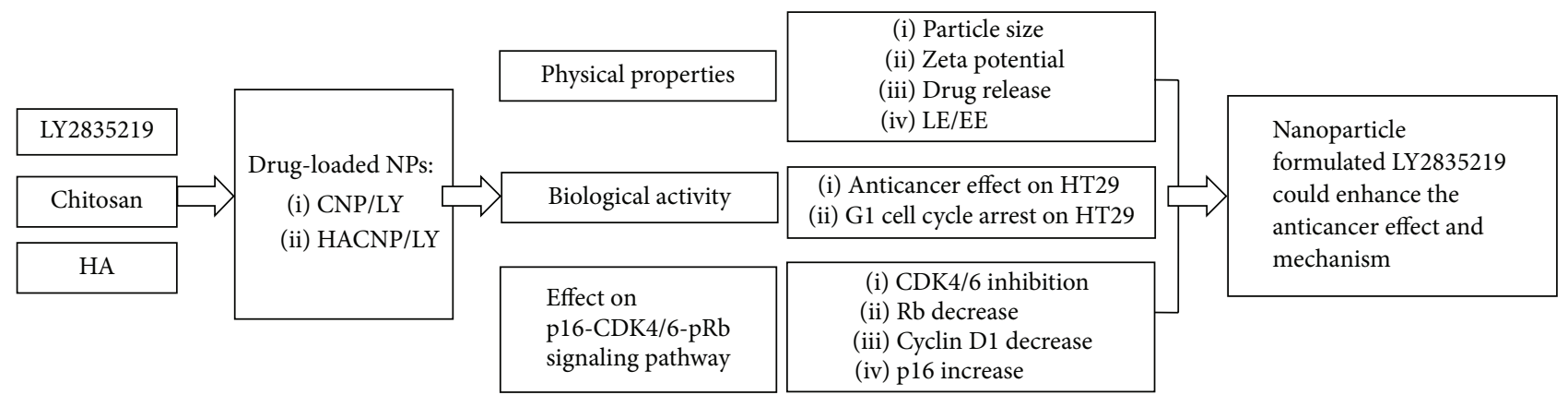

FIGURE 6: Schematic representation of anticancer effect and mechanism of LY2835219-loaded nanoparticles on HT29 cell line.

in 40 associated lymph node metastases. Cyclin D1 overexpression was seen in 55\% of cancers, pRb loss was in 33\%, and p16 loss was in 49\%. pRb expression was lower in lymph node metastases than in the corresponding primary tumors. Gongoll et al. found abnormal expression of p16 and Rb in tissues from 709 patients with colorectal cancer [27]. Von Stockmar-Von Wangenheim et al. reported that expressions of cyclin D1, p16, and Rb were abnormal in colorectal tissue samples [28]. Miladi-Abdennadher et al. [29] investigated an abnormal high expression of p16 and a positive correlation between p16 and metastasis in $38.6 \%$ of tissues from 70 intestinal cancer patients.

The in vivo anticancer effect was verified by Tate et al. [16], while there were few reports on the in vitro anticancer effect on colon cancer cells. We expected the present study to provide in vitro cell-based evidence. The protein levels of p16 and pRb were changed by LY2835219, which proved the feedback and interaction in p16-CDK4/6-pRb signal pathway.
And the result was consistent with the similar efficacy exerted by LEE011 and PD-0332991, two other CDK4/6 dual inhibitors. Treatment with LEE011 significantly reduced $\mathrm{pRb}$ expression in human neuroblastoma-derived cell lines [30]. Similarly, PD-0332991 induced inactivation of Rb in 16 glioblastoma multiforme cell lines. Another study revealed that low level of p16 caused more sensitivity to PD-0332991 in 40 ovarian cancer cell lines [31]. Additionally, level rise of p16 was observed, which inferred that a negative feedback of $\mathrm{Rb}$ protein to $\mathrm{p} 16$ protein presumably existed. In other types of tumor [32], researchers hypothesized that $\mathrm{pRb}$ family proteins negatively regulate p16 gene expression; in other words, when $\mathrm{pRb}$ protein is inactivated, expression of $\mathrm{p} 16$ increased.

As expected, nanoparticle formulations of LY2835219 induced more significant changes about these molecules, and HACNP/LY presented the strongest changes. It was speculated that the target delivery effect by chitosan and 
HA enhanced the delivery ability, leading to increasing the LY2835219 concentration intracellularly.

\section{Conclusion}

In conclusion, the anticancer effect and mechanism against colon cell line by LY2835219 and its nanoparticles DDS were observed for the first time. CDK4/6 inhibitor, LY2835219, not only delayed the cell growth but also affected the molecules in p16-cyclin D1/CDK4/6-pRb pathway (both upstream and downstream molecules) (Figure 6). Furthermore, the study was the first to evaluate the impact on p16-cyclin D1/CDK4/6$\mathrm{pRb}$ pathway by nanoparticle formulations and the increased impact derived from the affinity of chitosan and the HACD44 binding.

$\begin{array}{ll}\text { Abbreviations } \\ \text { CNP/LY: } & \text { LY2835219-loaded chitosan nanoparticles } \\ \text { HACNP/LY: } & \text { LY2835219-loaded HA-conjugated } \\ & \text { chitosan nanoparticles } \\ \text { CRC: } & \text { Colorectal cancer } \\ \text { EU: } & \text { The European Union } \\ \text { HA: } & \text { Hyaluronic acid } \\ \text { DDS: } & \text { Drug delivery system } \\ \text { NPs: } & \text { Nanoparticles } \\ \text { Rb: } & \text { Retinoblastoma } \\ \text { CKI: } & \text { Cyclin-dependent kinase inhibitor protein } \\ \text { NSCLC: } & \text { Non-small cell lung cancer } \\ \text { PBS: } & \text { Phosphate buffered saline } \\ \text { EE: } & \text { Encapsulation efficiency } \\ \text { LE: } & \text { Loading efficiency } \\ \text { qRT-PCR: } & \text { Real-time quantitative PCR. }\end{array}$

\section{Competing Interests}

The authors declare that there are no competing interests regarding the publication of this paper.

\section{Acknowledgments}

This work was supported by Grant no. 14ZRZD01 from Scientific Research Project of Sichuan College of Traditional Chinese Medicine and Grant no. 14S-01-3 from Scientific Research Plan of Mianyang Municipal Science \& Technology Bureau of Sichuan Province.

\section{References}

[1] M. Malvezzi, G. Carioli, P. Bertuccio et al., "European cancer mortality predictions for the year 2016 with focus on leukaemias," Annals of Oncology, vol. 27, no. 4, pp. 725-731, 2016.

[2] A. Gulbake, A. Jain, A. Jain, A. Jain, and S. K. Jain, "Insight to drug delivery aspects for colorectal cancer," World Journal of Gastroenterology, vol. 22, no. 2, pp. 582-599, 2016.

[3] M. Thanou, J. C. Verhoef, and H. E. Junginger, "Chitosan and its derivatives as intestinal absorption enhancers," Advanced Drug Delivery Reviews, vol. 50, no. 1, pp. S91-S101, 2001.
[4] M. Thanou, J. C. Verhoef, and H. E. Junginger, "Oral drug absorption enhancement by chitosan and its derivatives," Advanced Drug Delivery Reviews, vol. 52, no. 2, pp. 117-126, 2001.

[5] S. A. Agnihotri, N. N. Mallikarjuna, and T. M. Aminabhavi, "Recent advances on chitosan-based micro- and nanoparticles in drug delivery," Journal of Controlled Release, vol. 100, no. 1, pp. 5-28, 2004.

[6] M. S. Karbownik and J. Z. Nowak, "Hyaluronan: towards novel anti-cancer therapeutics," Pharmacological Reports, vol. 65, no. 5, pp. 1056-1074, 2013.

[7] C.-W. Lin, K.-Y. Lu, S.-Y. Wang, H.-W. Sung, and F.-L. Mi, "CD44-specific nanoparticles for redox-triggered reactive oxygen species production and doxorubicin release," Acta Biomaterialia, vol. 35, pp. 280-292, 2016.

[8] A. Jain and S. K. Jain, "Optimization of chitosan nanoparticles for colon tumors using experimental design methodology," Artificial Cells, Nanomedicine, and Biotechnology, 2015.

[9] B. Xiao, M. K. Han, E. Viennois et al., "Hyaluronic acid-functionalized polymeric nanoparticles for colon cancer-targeted combination chemotherapy," Nanoscale, vol. 7, no. 42, pp. 17745-17755, 2015.

[10] A. Jain, S. K. Jain, N. Ganesh, J. Barve, and A. M. Beg, "Design and development of ligand-appended polysaccharidic nanoparticles for the delivery of oxaliplatin in colorectal cancer," Nanomedicine, vol. 6, no. 1, pp. 179-190, 2010.

[11] A. Jain and S. K. Jain, "In vitro and cell uptake studies for targeting of ligand anchored nanoparticles for colon tumors," European Journal of Pharmaceutical Sciences, vol. 35, no. 5, pp. 404-416, 2008.

[12] A. Y. Chow, "Cell cycle control by oncogenes and tumor suppressors: driving the transformation of normal cells into cancerous cells," Nature Education, vol. 3, no. 9, article 7, 2010.

[13] M. Malumbres and M. Barbacid, "Mammalian cyclin-dependent kinases," Trends in Biochemical Sciences, vol. 30, no. 11, pp. 630-641, 2005.

[14] R. M. Feakins, C. D. Nickols, H. Bidd, and S.-J. Walton, "Abnormal expression of pRb, p16, and cyclin D1 in gastric adenocarcinoma and its lymph node metastases: relationship with pathological features and survival," Human Pathology, vol. 34, no. 12, pp. 1276-1282, 2003.

[15] E. Hamilton and J. R. Infante, "Targeting CDK4/6 in patients with cancer," Cancer Treatment Reviews, vol. 45, pp. 129-138, 2016.

[16] S. C. Tate, S. Cai, R. T. Ajamie et al., "Semi-mechanistic pharmacokinetic/pharmacodynamic modeling of the antitumor activity of LY2835219, a new cyclin-dependent kinase 4/6 inhibitor, in mice bearing human tumor xenografts," Clinical Cancer Research, vol. 20, no. 14, pp. 3763-3774, 2014.

[17] X. M. Wang and W. S. Liu, "The advance of applied research of chitosan and its derivatives in animal cell culture," Chinese Journal of Marine Drugs, vol. 19, no. 5, pp. 37-45, 2000.

[18] C. L. Liu, C. B. Li, and W. X. Cui, “The application of chitosan in medicine," Shandong Science, vol. 16, no. 3, pp. 68-71, 2003.

[19] S.-K. Kim and N. Rajapakse, "Enzymatic production and biological activities of chitosan oligosaccharides (COS): a review," Carbohydrate Polymers, vol. 62, no. 4, pp. 357-368, 2005.

[20] R. D. Price, M. G. Berry, and H. A. Navsaria, "Hyaluronic acid: the scientific and clinical evidence," Journal of Plastic, Reconstructive and Aesthetic Surgery, vol. 60, no. 10, pp. 11101119, 2007. 
[21] H. Acevedo, N. Gonzalez, and R. Moss, "Trophoblastic hormones and cancer: a breakthrough in treatment?" in Proceedings of the Comprehensive Cancer Care Conference, Session 205, June 1998.

[22] Q. M. Xu, L. C. Guo, X. H. Gu et al., "Prevention of colorectal cancer liver metastasis by exploiting liver immunity via chitosan-TPP/nanoparticles formulated with IL-12," Biomaterials, vol. 33, no. 15, pp. 3909-3918, 2012.

[23] B. Bánky, L. Rásó-Barnett, T. Barbai, J. Tímár, P. Becságh, and E. Rásó, "Characteristics of CD44 alternative splice pattern in the course of human colorectal adenocarcinoma progression," Molecular Cancer, vol. 11, article 83, 2012.

[24] Q. Gan, T. Wang, C. Cochrane, and P. McCarron, "Modulation of surface charge, particle size and morphological properties of chitosan-TPP nanoparticles intended for gene delivery," Colloids and Surfaces B: Biointerfaces, vol. 44, no. 2-3, pp. 6573, 2005.

[25] A. Nasti, N. M. Zaki, P. de Leonardis et al., "Chitosan/TPP and chitosan/TPP-hyaluronic acid nanoparticles: systematic optimisation of the preparative process and preliminary biological evaluation," Pharmaceutical Research, vol. 26, no. 8, pp. 19181930, 2009.

[26] B. M. Ku, S. Y. Yi, J. Koh et al., “The CDK4/6 inhibitor LY2835219 has potent activity in combination with mTOR inhibitor in head and neck squamous cell carcinoma," Oncotarget, vol. 7, no. 12, pp. 14803-14813, 2016.

[27] S. Gongoll, G. Peters, M. Mengel et al., "Prognostic significance of calcium-binding protein S100A4 in colorectal cancer," Gastroenterology, vol. 123, no. 5, pp. 1478-1484, 2002.

[28] C. A. S. Von Stockmar-Von Wangenheim, S. P. Mönig, P. M. Schneider et al., "p16, cyclin D1 and Rb expression in colorectal carcinomas: correlations with clinico-pathological parameters and prognosis," Molecular Medicine Reports, vol. 1, no. 1, pp. 2732, 2008.

[29] I. Miladi-Abdennadher, R. Abdelmaksoud-Damak, L. Ayadi et al., "Expression of p16INK4a, alone or combined with p53, is predictive of better prognosis in colorectal adenocarcinoma in Tunisian patients," Applied Immunohistochemistry and Molecular Morphology, vol. 19, no. 6, pp. 562-568, 2011.

[30] J. Rader, M. R. Russell, L. S. Hart et al., "Dual CDK4/CDK6 inhibition induces cell-cycle arrest and senescence in neuroblastoma," Clinical Cancer Research, vol. 19, no. 22, pp. 6173-6182, 2013.

[31] G. E. Konecny, B. Winterhoff, T. Kolarova et al., "Expression of p16 and retinoblastoma determines response to CDK4/6 inhibition in ovarian cancer," Clinical Cancer Research, vol. 17, no. 6, pp. 1591-1602, 2011.

[32] InTech, Human, Rosario Guerrero Tejada, 2012. 

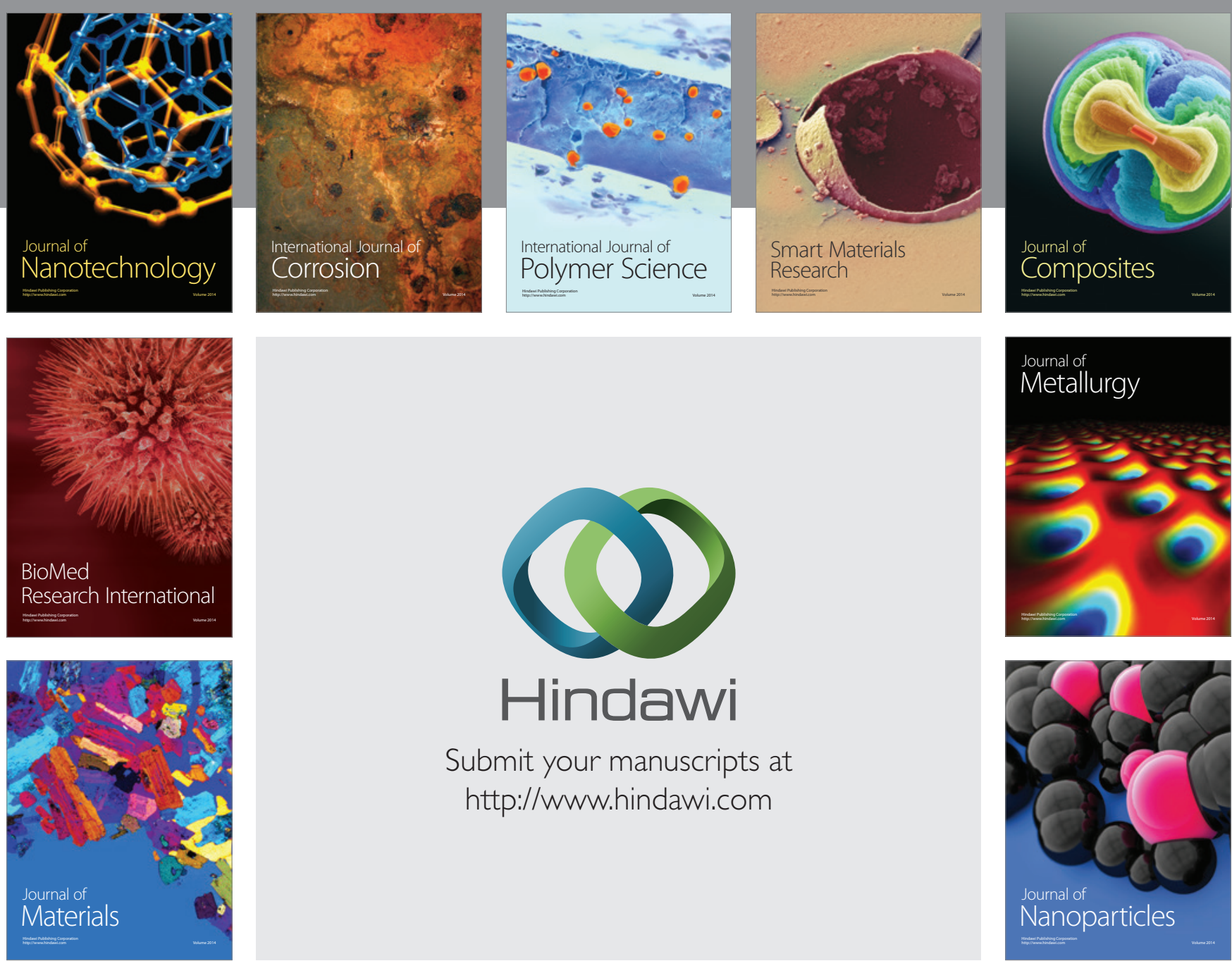

\section{Hindawi}

Submit your manuscripts at

http://www.hindawi.com

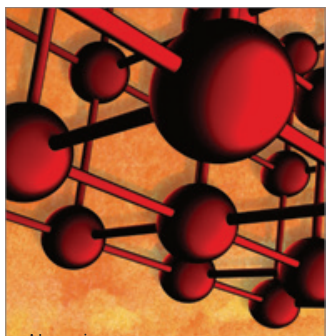

Materials Science and Engineering
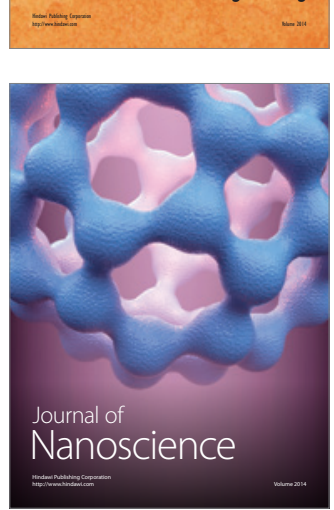
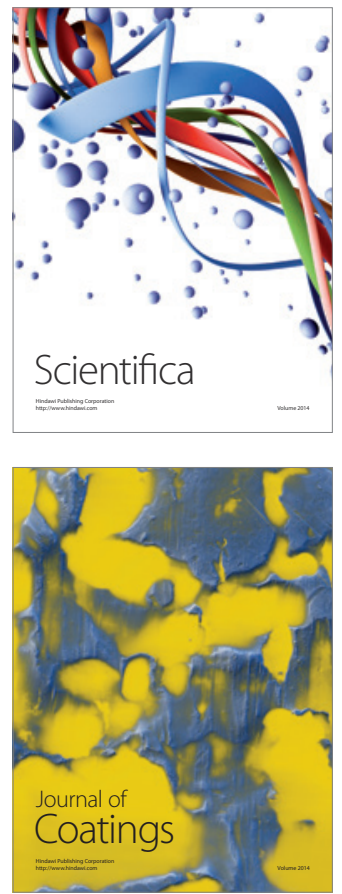
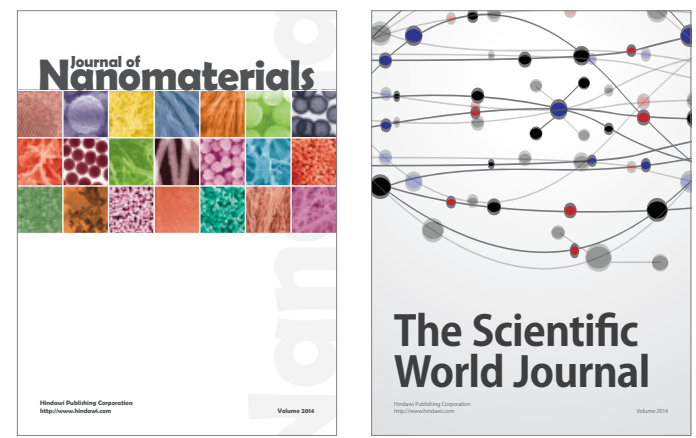

The Scientific World Journal
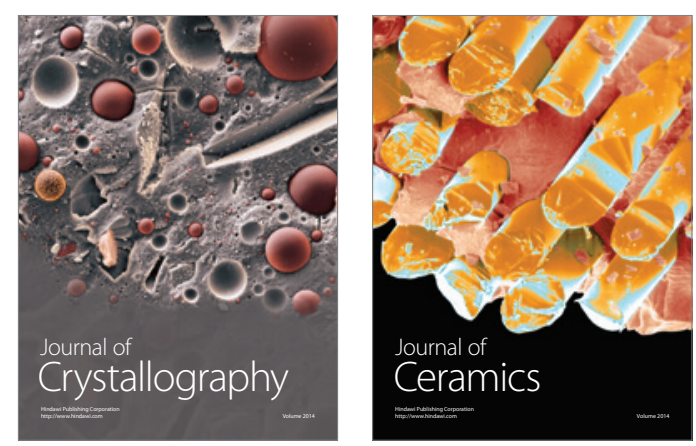
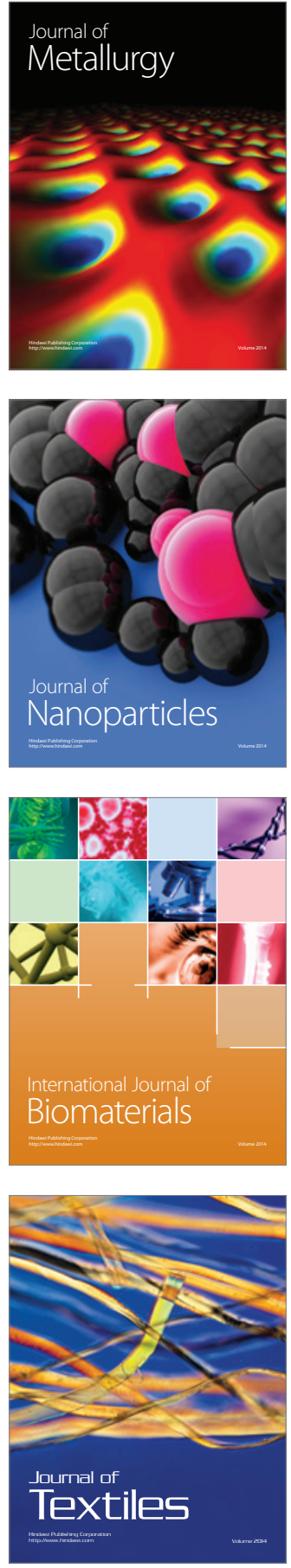